\title{
STAT4 and STAT6 regulate systemic inflammation and protect against lethal endotoxemia
}

\author{
Alex B. Lentsch, Atsushi Kato, Brian Davis, Warner Wang, Celia Chao, \\ and Michael J. Edwards \\ Department of Surgery, University of Louisville School of Medicine, Louisville, Kentucky, USA \\ Address correspondence to: Alex B. Lentsch, Department of Surgery, University of Louisville, \\ James Graham Brown Cancer Center, Room 426, Louisville, Kentucky 40202, USA. \\ Phone: (502) 852-7698; Fax: (502) 852-2975; E-mail: alentsch@louisville.edu. \\ Received for publication July 16, 2001, and accepted in revised form September 25, 2001.
}

\begin{abstract}
Members of the signal transducer and activator of transcription (STAT) family are transcription factors that mediate many of the effects of pro- and anti-inflammatory cytokines. The progressive systemic inflammatory response induced by endotoxin is mediated by overzealous cytokine production. Here we identify STAT4 and STAT6 as critical regulators of the systemic inflammatory response to endotoxin. Mice deficient for STAT4 or STAT6 were highly susceptible to lethal endotoxemia. In STAT4 $4^{-/-}$mice, antibody blockade of IL-12 prevented mortality, suggesting that STAT4 confers protection, while another signaling pathway mediates the detrimental effects of IL-12. In STAT6 ${ }^{-/-}$mice we observed dysregulated activation of the transcription factor NF- $\kappa \mathrm{B}$, resulting in augmented production of proinflammatory cytokines and chemokines. Furthermore, STAT6 $6^{-/-}$mice displayed increased organ accumulation of leukocytes and significant hepatocellular injury. These findings demonstrate that STAT4 and STAT6 confer protection against endotoxin-induced death and that for STAT6 these protective effects occur through the regulation of NF- $\mathrm{KB}$ activation and subsequent production of proinflammatory cytokines and chemokines.
\end{abstract}

J. Clin. Invest. 108:1475-1482 (2001). DOI:10.1172/JCI200113763.

\section{Introduction}

The septic state is associated with a generalized activation of inflammatory pathways following infection. In Gram-negative infections endotoxin is a primary stimulus of the inflammatory response. Endotoxin binds to a variety of cell types, including macrophages, monocytes, and endothelial cells, triggering activation of the transcription factor NF- $\mathrm{NB}(1)$. Activation of $\mathrm{NF}-\kappa \mathrm{B}$, which consists of nuclear translocation and DNA binding, results in the expression of proinflammatory cytokines, chemokines, and VCAMs $(2,3)$. During sepsis or endotoxemia, the dysregulated production of these mediators may result in an overwhelming systemic inflammatory response that contributes to disturbed cardiopulmonary function and hemodynamic instability, sometimes progressing to multiorgan failure and death (4-6). While NF- $\mathrm{BB}$ appears to be the primary endotoxin-induced transcriptional activator of proinflammatory mediators, little is known about the transcriptional control mechanisms that attempt to regulate the inflammatory response during endotoxemia.

The signal transducer and activator of transcription (STAT) family of transcription factors includes seven members that are activated in response to different cytokine-receptor interactions (7). Of this family, STAT4 and STAT6 are highly relevant to the inflammatory response to bacteria or endotoxin. STAT4 is the primary transcription factor utilized by $\operatorname{IL}-12(8,9)$. IL-12 is critical for bacterial containment and clearance in septic peritonitis and for promotion of the inflammatory response during endotoxemia (10-12). STAT6 is activated by IL-4 and IL-13 (13-15). No role for IL-4 has been established in either sepsis or endotoxemia, but IL-13 appears to be a major regulator of the inflammatory response of both models by preventing organ dysfunction and death $(16,17)$. In the cecal ligation and puncture model of septic peritonitis, mice lacking either STAT4 or STAT6 had less organ injury and mortality, suggesting that STAT4 and STAT6 are intimately involved in the host response to infection (18).

The objective of the current study was to investigate the roles of STAT4 and STAT6 in the systemic inflammatory response induced by endotoxin. Since this model is devoid of any infectious burden, it provides information strictly related to the systemic inflammatory response induced by this component of Gram-negative bacteria. Our results demonstrate that mice lacking either STAT4 or STAT6 are much more susceptible to lethal endotoxemia than their wild-type counterparts. Furthermore, blockade of endogenous IL-12 in STAT4 ${ }^{-1-}$ mice prevented the lethal effects of endotoxin, suggesting that the deleterious effects of IL-12 are mediated by another signaling pathway. In STAT6-/mice, there was unregulated activation of NF- $\kappa \mathrm{B}$ and increased chemokine expression in liver and lung. These findings suggest that STAT4 and STAT6 regulate the systemic inflammatory response to endotoxin by distinct mechanisms. 


\section{Methods}

Murine model of endotoxemia. STAT4 ${ }^{-/}$and STAT6 ${ }^{-/-}$mice (backcrossed ten generations to $\mathrm{BALB} / \mathrm{c}$ ) were purchased from The Jackson Laboratory (Bar Harbor, Maine, USA) and bred and maintained at the University of Louisville. Age- and sex-matched BALB/c mice were purchased from The Jackson Laboratory and used as wild-type controls. For induction of endotoxemia, mice received 3 $\mathrm{mg} / \mathrm{kg}$ endotoxin (LPS from Escherichia coli, serotype 0111:B4; Sigma Chemical Co., St. Louis, Missouri, USA) in $0.75 \mathrm{ml}$ PBS by intraperitoneal injection. Control mice received $0.75 \mathrm{ml}$ PBS. For IL-12 blockade, $300 \mu \mathrm{g}$ of affinity-purified polyclonal rabbit anti-mouse IL-12 IgG was administered via the tail vein just prior to endotoxin injection. We have previously shown that this dose of anti-IL-12 effectively neutralizes the biological activity of IL-12 in a murine model of hepatic ischemia/reperfusion (19). Controls received $300 \mu \mathrm{g}$ of preimmune rabbit IgG. This project was approved by the University of Louisville Animal Care and Use Committee and was in compliance with $\mathrm{NIH}$ guidelines.

Immunoprecipitation and Western blot. Liver and lung tissues were homogenized in cold PBS supplemented with $1 \%$ NP-40, $0.5 \%$ deoxycholate, $0.1 \%$ SDS, $1 \mu \mathrm{g} / \mathrm{ml}$ leupeptin, $1 \mu \mathrm{g} / \mathrm{ml}$ aprotonin, $10 \mu \mathrm{g} / \mathrm{ml}$ soybean trypsin inhibitor, $1 \mu \mathrm{g} / \mathrm{ml}$ pepstatin, and $100 \mu \mathrm{g} / \mathrm{ml}$ of PMSF. Protein concentration was determined by bicinchoninic acid protein assay (Pierce Chemical Co., Rockford, Illinois, USA). One milligram of total protein was used for immunoprecipitation. The protein samples were precleared with $200 \mu \mathrm{l}$ of protein G (Life Technologies Inc., Rockville, Maryland, USA) per sample for 1 hour at $4^{\circ} \mathrm{C}$. Antibodies to STAT4 and STAT6 (Santa Cruz Biotechnology Inc., Santa Cruz, California, USA) were prebound to protein $\mathrm{G}$ and $2 \mu \mathrm{g}$ antibody/protein $\mathrm{G}$ conjugate was added to each sample and incubated for 2 hours at $4^{\circ} \mathrm{C}$. The beads were washed three times with cold supplemented PBS, resuspended in SDS sample buffer, and boiled for 3 minutes. The immunoprecipitated samples were separated by $10 \%$ SDS-PAGE and transferred to a PVDF membrane. The membranes were blocked in 5\% BSA in Tris-buffered saline overnight at $4{ }^{\circ} \mathrm{C}$ and reblocked in 5\% milk in Tris-buffered saline plus $0.2 \%$ Tween-20 (TTBS) for 30 minutes at room temperature. Mouse anti-phosphotyrosine antibody (Santa Cruz Biotechnology Inc.) was diluted at 1:1000 with 5\% milk in TTBS and incubated with the membranes for 1 hour at room temperature. The membranes were washed three times with TTBS and incubated with goat anti-mouse IgG horseradish peroxidase secondary antibody (Santa Cruz Biotechnology Inc.), diluted at 1:5000 with 5\% milk in TTBS, for 30 minutes at room temperature. The membranes were washed as above and immunoreactive proteins were visualized using enhanced chemiluminescence. Electrophoretic mobility shift assay. Nuclear extracts of liver tissue were prepared by the method of Deryckere and Gannon (20) and analyzed by electrophoretic mobility shift assay (EMSA). Briefly, double-stranded NF- $\mathrm{KB}$ consensus oligonucleotide (Promega Corp., Madison, Wis- consin, USA) was end-labeled with $\gamma\left[{ }^{32} \mathrm{P}\right]$ ATP $(3,000$ $\mathrm{Ci} / \mathrm{mmol}$ at $10 \mathrm{mCi} / \mathrm{ml}$; Amersham Life Sciences Inc., Arlington Heights, Illinois, USA). Binding reactions containing equal amounts of nuclear protein extract $(20 \mu \mathrm{g})$ and $35 \mathrm{fmol}(\sim 50,000 \mathrm{cpm}$, Cherenkov counting) of oligonucleotide were incubated at room temperature for 30 minutes. Reaction volumes were held constant to 15 $\mu l$. Reaction products were separated in a native $4 \%$ polyacrylamide gel and analyzed by autoradiography.

Ribonuclease protection assay. Total RNA from lung and liver was extracted with TRIzol Reagent (Life Technologies Inc.) Ribonuclease protection assays were performed using RiboQuant kits purchased from Pharmingen (San Diego, California, USA) as described by the manufacturer. A customized DNA template was used to analyze the expression of the chemokines lymphotactin (Ltn), RANTES, eotaxin, macrophage inflammatory protein-1 $\alpha$ (MIP- $1 \alpha)$, MIP- $1 \beta$, MIP- 2 , IFN- $\gamma$ inducible protein-10 (IP-10), and monocyte chemoattractant protein-1 (MCP-1), as well as the housekeeping gene GAPDH. The probes were hybridized with $10 \mu \mathrm{g}$ of total RNA for 16 hours at $56^{\circ} \mathrm{C}$, after which free probe and other single-stranded RNA was digested with RNases. Double-stranded (protected) RNA was purified and resolved using the QuickPoint Gel system (Novex, San Diego, California, USA).

Serum and tissue cytokine/chemokine assays. ELISA reagents for TNF- $\alpha$, MIP-2, MCP-1, IFN- $\gamma$, IL-6, and GM-CSF were from PeproTech Inc. (Rocky Hill, New Jersey, USA). ELISA reagents for IL-10 and IL-13 were purchased from R\&D Systems Inc. (Minneapolis, Minnesota, USA), and reagents for IL-12 were from Pharmingen. Extraction of proteins from tissue samples for ELISA analyses was performed as described elsewhere (21). Briefly, tissues were weighed and immediately placed in 10 volumes (wt/vol) of a protease inhibitor cocktail containing $10 \mathrm{mM}$ EDTA, $2 \mathrm{mM}$ PMSF, $0.1 \mathrm{mg} / \mathrm{ml}$ soybean trypsin inhibitor, $1.0 \mathrm{mg} / \mathrm{ml}$ BSA, $1 \%$ penicillin-streptomycin, and $0.02 \%$ sodium azide in isotonic PBS, pH 7.0. Tissues were disrupted with a tissue homogenizer and lysates were incubated at $4^{\circ} \mathrm{C}$ for 2 hours. Samples were clarified by two rounds of centrifugation at $12,500 \mathrm{~g}$ for 10 minutes at $4^{\circ} \mathrm{C}$. Protein concentration was determined by bicinchoninic

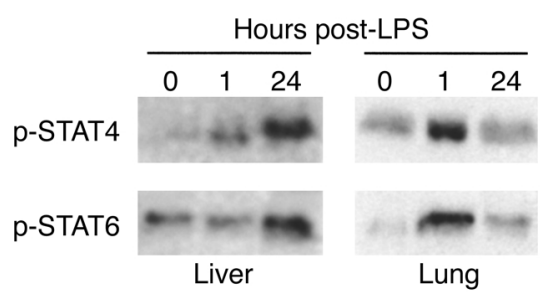

\section{Figure 1}

Activation of STAT4 and STAT6 in liver and lung tissues during endotoxemia. STAT4 and STAT6 were immunoprecipitated from $1 \mathrm{mg}$ of homogenates of liver and lung harvested at time 0 or 1 or 24 hours after administration of $3 \mathrm{mg} / \mathrm{kg}$ endotoxin. Immunoprecipitates were separated by SDS-PAGE, and Western blots were stained with antiphosphotyrosine. 


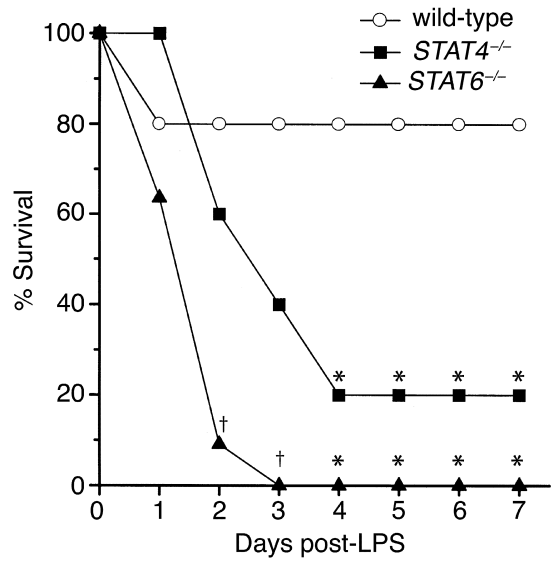

Figure 2

STAT4 $4^{-/}$and STAT6- ${ }^{--}$mice are more susceptible to lethal endotoxemia than wild-type mice. Following intraperitoneal administration of $3 \mathrm{mg} / \mathrm{kg}$ endotoxin, wild-type, STAT4 ${ }^{-/-}$, and STAT6 ${ }^{-/-}$mice were monitored for survival. Those mice alive at day 7 were alive at day 15 (data not shown). For each group, $n=10-11 .{ }^{*} P<0.05$ compared with wildtype mice; ${ }^{\dagger} P<0.05$ compared with wild-type and STAT4 ${ }^{-1-}$ mice.

acid assay with trichloroacetic acid precipitation using BSA as a reference standard (Pierce Chemical Co.).

Histology and clinical chemistry. Tissues were fixed in $10 \%$ buffered formalin, embedded in paraffin, sectioned, and stained with hematoxylin and eosin. Twenty high-power fields per tissue section were observed for each animal, and the number of granulocytes and mononuclear cells was recorded. Data are expressed as the number of cells per high-power field. Serum was analyzed for alanine aminotransferase (ALT) as an index of liver injury. Measurements of serum ALT were made using a diagnostic kit from Sigma Chemical Co. Myeloperoxidase assay. Liver myeloperoxidase (MPO) content was assessed by methods similar to those of Schierwagen et al. (22). Liver tissue $(100 \mathrm{mg})$ was homogenized in $2 \mathrm{ml}$ of buffer $\mathrm{A}\left(3.4 \mathrm{mM} \mathrm{KH}_{2} \mathrm{HPO}_{4}\right.$, $16 \mathrm{mM} \mathrm{Na}_{2} \mathrm{HPO}_{4}, \mathrm{pH}$ 7.4). After centrifugation for 20 minutes at $10,000 \mathrm{~g}$, the pellet was resuspended in 10 volumes of buffer B $\left(43.2 \mathrm{mM} \mathrm{KH}_{2} \mathrm{HPO}_{4}, 6.5 \mathrm{mM}\right.$ $\mathrm{Na}_{2} \mathrm{HPO}_{4}, 10 \mathrm{mM}$ EDTA, $0.5 \%$ hexadecyltrimethylammonium, $\mathrm{pH}$ 6.0) and sonicated for 10 seconds. After heating for 2 hours at $60^{\circ} \mathrm{C}$, the supernatant was reacted with 3,3', 3,5'-tetramethylbenzidine (Sigma Chemical Co.) and optical density determined at $655 \mathrm{~nm}$.

Statistical analysis. All data are expressed as mean \pm SEM. Survival data were analyzed with Fisher's exact test. All other data were analyzed with a one-way ANOVA with subsequent Student-Newman-Keuls test. Differences were considered significant at $P<0.05$.

\section{Results}

STAT4 and STAT6 are activated in liver and lung during endotoxemia. Both IL-12 and IL-13 have been shown to be critically important to the systemic inflammatory response to endotoxin $(10,16)$, suggesting potential roles for STAT4 and STAT6. In order to assess whether endotoxin resulted in activation of STAT4 and STAT6 in major organs, we assessed the level of activated (phosphorylated) STAT4 and STAT6 in liver and lung. Figure 1 shows the results of immunoprecipitation/Western blot analysis of phosphorylated STAT4 and STAT6 in liver and lung. In liver, little activated STAT4 was observed at time 0. STAT6 appeared to have a basal level of constitutive activation. One hour after endotoxin, levels of phosphorylated STAT4, but not STAT6, were increased. However, 24 hours after endotoxin there was a dramatic increase in the amount of phosphorylated STAT4 and STAT6 in liver extracts. In lung, phosphorylated forms of both STAT4 and STAT6 were increased 1 hour after endotoxin. After 24 hours, the amount of phosphorylated STAT4 resembled that at time 0 , while levels of phosphorylated STAT6 remained above that of time 0 . Thus, it appears that activation of STAT4 and STAT6 occurs in distinct organ-specific, time-dependent fashions.

STAT4 and STAT6 are required for protection against lethal endotoxemia. To determine whether activation of STAT4 and STAT6 are important regulatory events in the response to endotoxin, we compared the survival rates of wild-type, STAT4 $4^{-/}$, and STAT6 ${ }^{-/-}$mice after administration of $3 \mathrm{mg} / \mathrm{kg}$ endotoxin. Twenty percent of wildtype mice died within 24 hours, but none died thereafter, yielding a survival rate of $80 \%$ (Figure 2). Forty percent of $S T A T 4^{-/}$mice died within 2 days, $60 \%$ died after 3 days, and $80 \%$ were dead after 4 days $(P=0.023)$. No STAT4 $4^{-/}$mice died after 4 days, resulting in a $20 \%$ survival rate. STAT6 ${ }^{-/}$mice died precipitously after endotoxin, with $36 \%$ dead after 24 hours, $91 \%$ dead after 2 days $(P=0.002)$, and $100 \%$ dead by 3 days $(P<0.001)$. For all mice (wild-type, STAT4 $4^{--}$, and STAT6 ${ }^{-/-}$), those surviving after 7 days were alive after 15 days. Mortality was significantly greater in $S T A T 6^{-/}$mice than in STAT4 ${ }^{--}$mice 2 days $(P=0.024)$ and 3 days $(P=0.035)$ after endotoxin (Figure 2). These data demonstrate that

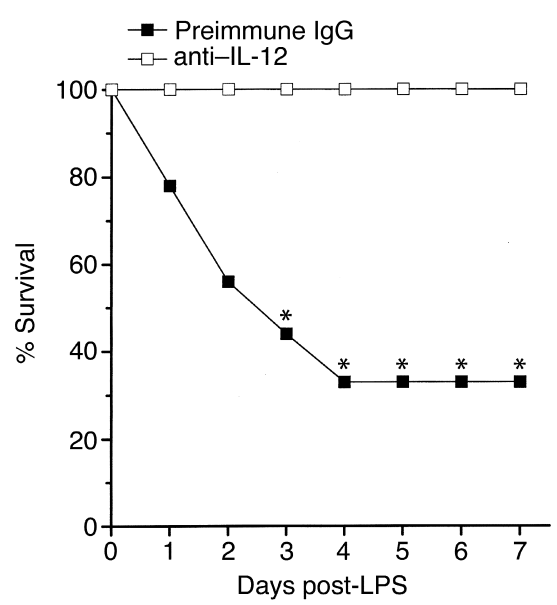

Figure 3

Effects of anti-IL-12 on endotoxin lethality in STAT4 ${ }^{-/-}$mice. Survival was assessed in STAT4 ${ }^{-1-}$ mice receiving $300 \mu \mathrm{g}$ preimmune rabbit IgG or $300 \mu \mathrm{g}$ anti-IL-12 intravenously just prior to intraperitoneal administration of $3 \mathrm{mg} / \mathrm{kg}$ endotoxin. Those mice alive at day 7 were alive at day 15 (data not shown). For each group, $n=9$. ${ }^{*} P<0.05$ compared with mice receiving preimmune lgG. 


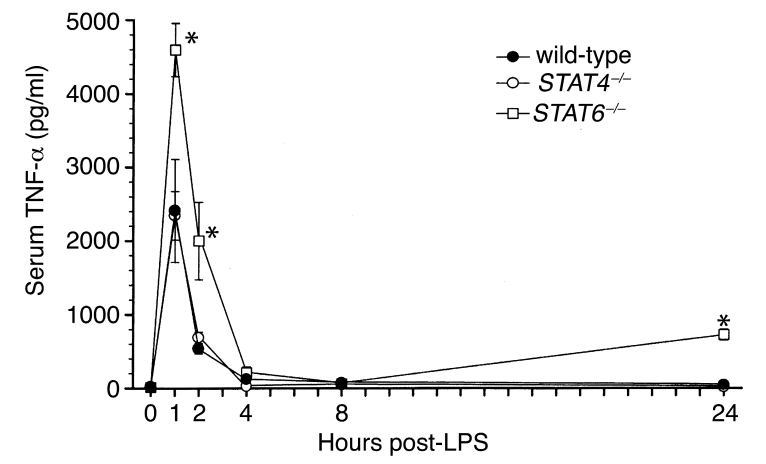

Figure 4

Serum levels of TNF- $\alpha$ during endotoxemia. TNF- $\alpha$ content in serum samples was measured by ELISA in wild-type, STAT4 ${ }^{-1-}$, and STAT6 ${ }^{-1-}$ mice after intraperitoneal injection of $3 \mathrm{mg} / \mathrm{kg}$ endotoxin. Values are mean \pm SEM with $n=5-8$ mice per group. ${ }^{*} P<0.05$ compared with wild-type and STAT4 ${ }^{-1-}$ mice.

STAT4- and STAT6-deficient mice are far more susceptible to lethal endotoxemia than wild-type mice.

Because STAT4 mediates many of the biological effects of IL-12 $(8,9)$, and blockade of IL-12 has been shown to be protective in a similar murine model of endotoxemia (10), we sought to determine whether, in this model, IL-12 has effects mediated by other signal transduction mechanisms. For these experiments, STAT4 $4^{-/-}$mice were treated with either $300 \mu \mathrm{g}$ preimmune rabbit $\mathrm{IgG}$ or anti-IL-12 just prior to endotoxin administration. As shown in Figure 3, none of the STAT4 ${ }^{-/}$mice receiving anti-IL-12 died, while $67 \%$ of those receiving preimmune IgG died within 4 days $(P=0.005)$. These data suggest that IL-12 contributes to the lethal effects of endotoxin by a STAT4-independent mechanism.

Augmented cytokine and chemokine expression in STAT6 ${ }^{-1-}$ mice. Endotoxemia is characterized by a rapid increase in the expression of the proinflammatory cytokine TNF- $\alpha$, as well as the subsequent multiorgan expression of CXC and CC chemokines (23-26). To investigate the potential mechanisms of the increased mortality observed in STAT4 ${ }^{-/}$and STAT6 ${ }^{-/-}$mice, we measured serum levels of TNF- $\alpha$ and expression of chemokine mRNA and protein in liver and lung. Wild-type mice showed the expected rapid increase in serum TNF- $\alpha$, with peak levels occurring 1 hour after endotoxin (Figure 4). STAT4 ${ }^{-/}$mice had a nearly identical pattern of TNF- $\alpha$ production. In STAT6 ${ }^{-/-}$ mice, however, serum TNF- $\alpha$ was greatly increased at 1 hour, 2 hours, and 24 hours, compared with both wildtype and STAT4 ${ }^{-/}$mice (Figure 4), indicating dysregulated production of this inflammatory cytokine.

A number of other cytokines have been implicated in the positive and negative regulation of systemic inflammation induced by endotoxemia. In order to ascertain whether the susceptibility of STAT4-/- and STAT6 ${ }^{-/-}$may be a result of altered tissue expression of pro- and/or antiinflammatory cytokines, we measured the expression of the proinflammatory cytokines IL-12, IFN- $\gamma$, and GM-CSF and the anti-inflammatory cytokines, IL- 6 , IL-10, and IL-13 in the liver and lung by ELISA 1 hour and 24 hours after endotoxin administration. With the excep- tion of IL-6, which was increased in liver and lungs of STAT6 ${ }^{-/-}$mice after 24 hours (Table 1 ), we found no differences in the protein expression of these mediators in wild-type, STAT4 $4^{--}$, or STAT6 ${ }^{-/}$mice at either time point.

Because the multi-organ leukocyte recruitment observed during endotoxemia is, in part, dependent upon increased organ expression of chemokines, we assessed the expression of chemokine mRNAs in liver and lung tissues. STAT4-/ mice differed from wild-type mice, with increased IP-10 and MCP-1 mRNA expression in liver 1 hour after endotoxin (Figure 5). Similarly, liver expression of IP-10 and MCP-1 mRNA was increased in livers of $S$ SAT6 $^{-/-}$mice after 1 hour. Twenty-four hours after endotoxin, chemokine mRNA expression in STAT4-/mice appeared identical to that of wild-type mice. However, $S T A T 6^{-/}$mice displayed greatly increased expression of mRNA for MIP-1 $\alpha$, MIP-2, IP-10, and MCP-1 in both liver and lung 24 hours after endotoxin (Figure 5). Measurement of MIP- $1 \alpha$, MIP-2, and MCP- 1 proteins in liver and lung 24 hours after endotoxin showed no differences between wild-type mice and STAT4 ${ }^{--}$mice (Figure 6). STAT6 $^{-/-}$mice showed increases in MIP-1 $\alpha$, MIP-2, and MCP-1 proteins in liver and MIP- $1 \alpha$ and MCP- 1 in lung. Thus, STAT6, but not STAT4, appears to be required for the regulation of cytokine and chemokine production during endotoxemia.

Activation of NF- $\mathrm{KB}$ is dysregulated in STAT6 ${ }^{-/}$mice. The transcription factor NF- $\mathrm{KB}$ has been implicated in the development of the systemic inflammatory response in sepsis and endotoxemia $(27,28)$. NF- $\mathrm{KB}$ is a major transcriptional regulator of numerous proinflammatory mediators, including TNF- $\alpha$, MIP- $1 \alpha$, MIP-2, and MCP-1 (3). In order to assess whether the function of $\mathrm{NF}-\mathrm{KB}$ was altered in $S T A T 6^{-/}$mice as a potential mechanism for the unregulated production of cytokines and chemokines, we measured the nuclear translocation and DNA binding of NF- $\mathrm{KB}$ in liver and lung extracts. In wild-type and STAT4-- mice, there was little NF- $\mathrm{KB}$ in nuclear extracts of liver and lung at time 0 (Figure 7). One hour after endotoxin, NF- $\mathrm{KB}$ activation was dramatically increased, returning to baseline levels after 24 hours. In contrast, NF- $\kappa B$ activation in liver and lungs of STAT6 $6^{--}$mice showed the rapid activation of NF- $\mathrm{KB}$ after 1 hour, but this high level of activation was also present 24 hours after endotoxin (Figure 7), suggesting that STAT6 is critical for the regulation of NF-KB activation and subsequent production of cytokines and chemokines during endotoxemia.

\section{Table 1}

IL-6 expression 24 hours after endotoxin administration

\begin{tabular}{lcc}
\multicolumn{3}{c}{ IL-6 $(\mathrm{pg} / \mathrm{mg}$ protein $)$} \\
& Liver & Lung \\
Wild-type & $59.6 \pm 3.2$ & $42.3 \pm 13.9$ \\
STAT4 ${ }^{--}-$ & $57.5 \pm 3.0$ & $37.6 \pm 5.5$ \\
STAT6 $^{--}$ & $114.8 \pm 16.3^{\mathrm{A}}$ & $251.0 \pm 49.8^{\mathrm{A}}$ \\
\cline { 2 - 2 }${ }^{\text {AP }}<0.05$ compared with wild-type and STAT4-/- mice. $n=4$ per group.
\end{tabular}




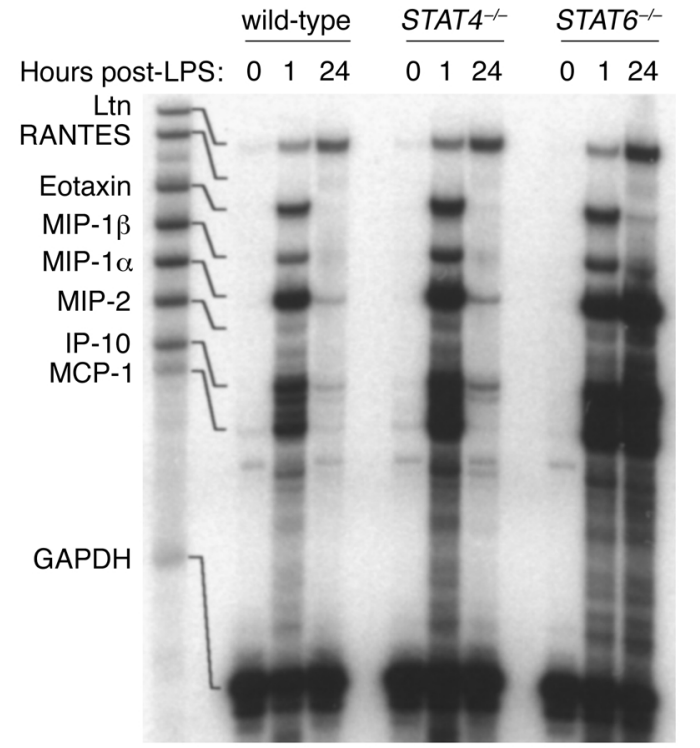

Liver

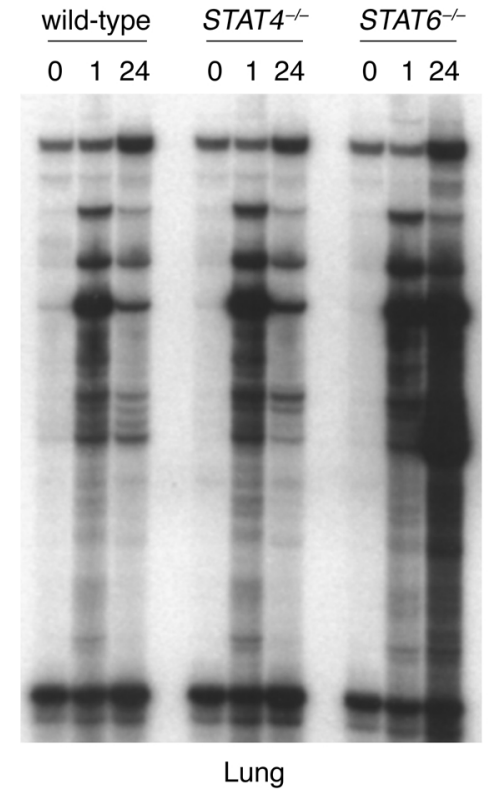

\section{Figure 5}

Chemokine mRNA expression in liver and lung tissues during endotoxemia. mRNA for the chemokines Ltn, RANTES, eotaxin, MIP- $1 \alpha$, MIP- $1 \beta$, MIP-2, IP-10, and MCP-1 were evaluated by ribonuclease protection assay. GAPDH was used to confirm the equality of in vitro transcription efficiency and sample loading. The first lane contains unprotected probes and the corresponding lines indicate the location of the protected mRNA species.
Increased hepatocellular damage and organ leukocyte accumulation in STAT6 ${ }^{-1-}$ mice. Endotoxin causes the accumulation of leukocytes in multiple organs. Therefore, we conducted experiments to determine whether the augmented production of cytokines and chemokines in STAT6 ${ }^{-/-}$mice was associated with increased leukocyte trafficking and organ dysfunction. Sections of liver and lung were evaluated for histopathological changes 24 hours after endotoxin. No differences were observed in lung sections from wild-type, STAT4 ${ }^{--}$, and STAT6 ${ }^{-/-}$mice; all showed similar thickening of the alveolar membranes (data not shown). In liver sections, tissue architecture appeared relatively normal in wild-type and STAT4 ${ }^{-1-}$ mice (Figure 8, a and b, respectively). However, livers from $S T A T 6^{-1-}$ mice showed abundant areas of hepatocyte necrosis (Figure 8c). These histopathological changes in the livers of $S T A T 6^{-/}$mice were associated with a significant increase in serum ALT, a marker of hepatocellular injury (Figure 8d). There were no changes in serum ALT levels in either wild-type or STAT4 ${ }^{-1-}$ mice. Thus, the greater susceptibility of STAT6 ${ }^{-/-}$ mice to the lethal effects of endotoxin may be related to the hepatocellular injury observed in these mice.
In both liver and lung sections, we observed large numbers of granulocytic and monocytic leukocytes. In lungs, these cells appeared to be adherent to the vascular endothelium, and there was no evidence of cells in the alveolar spaces. In liver, these cells were localized primarily along the vascular walls of central veins and within sinusoids. To determine whether there were differences in the extent of leukocyte accumulation in liver and lung between wild-type, STAT4-/-, and $S T A T 6^{-/-}$mice, we performed MPO assays (for quantitation of neutrophils) and morphometric analyses of granulocytes and mononuclear cells within these tissues. The results of our MPO assays are shown in Figure 9. In liver, no significant changes in MPO activity were observed 2, 4, or 8 hours after endotoxin (Figure 9a). After 24 hours, liver MPO levels in STAT6 ${ }^{-/-}$mice were up to threefold higher than the levels in wild-type and STAT4 ${ }^{-/}$mice, which were themselves increased above baseline. In lung, MPO activity in STAT4 ${ }^{-/-}$mice was significantly greater than in wild-type mice 1 and 2 hours after endotoxin, remained elevated after 8 hours, and decreased by 24 hours (Figure 9b). MPO lev-
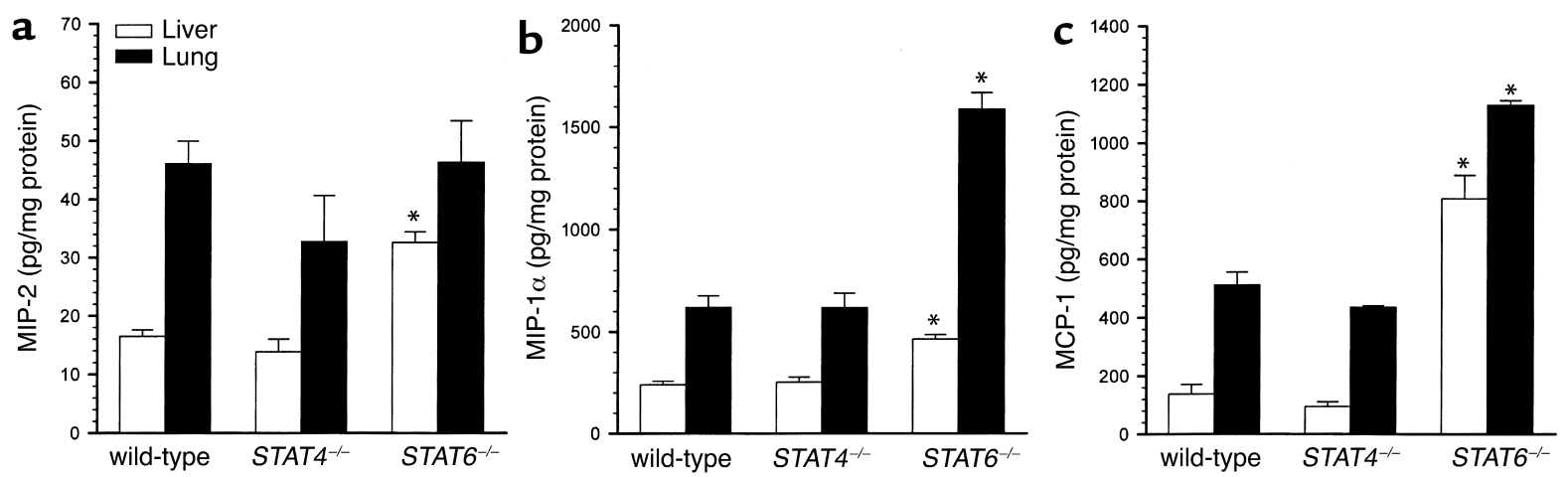

Figure 6

Chemokine protein expression in liver and lung 24 hours after endotoxin. Liver and lung homogenates were analyzed by ELISA for the chemokines MIP-2 (a), MIP-1 $\alpha(\mathbf{b})$, and MCP-1 (c). Values are mean \pm SEM with $n=6$ mice per group. ${ }^{*} P<0.05$ compared with wild-type controls. 

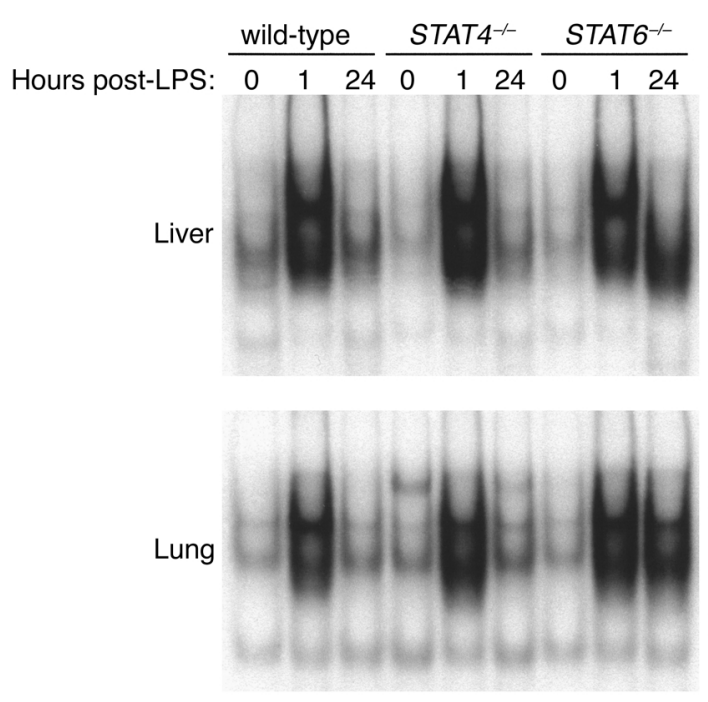

\section{Figure 7}

Activation of NF-KB during endotoxemia in wild-type, STAT4 ${ }^{-/-}$, and STAT6 ${ }^{-1-}$ mice. Liver and lung nuclear extracts obtained at time 0 or 1 or 24 hours after administration of $3 \mathrm{mg} / \mathrm{kg}$ endotoxin were analyzed by EMSA.

els in lungs from $\mathrm{STAT6}^{-/-}$mice were indistinguishable from those in wild-type mice for up to 8 hours, but 24 hours after endotoxin they were nearly threefold higher than in wild-type or STAT4 ${ }^{-/-}$mice. Based on these findings it appears that STAT4-/- mice have augmented neutrophil accumulation in lungs shortly after endotoxin but, by 24 hours, have similar amounts of neutrophils to those of wild-type mice. Conversely, $\mathrm{STAT6}^{-/-}$mice appear to have a delayed but grossly increased accumulation of neutrophils in liver and lung following endotoxin.

In addition to tissue MPO assays, we performed morphometric analyses to quantitate the number of granulocytes and mononuclear cells in liver and lung tissues 24 hours after endotoxin. As shown in Figure 6, livers from STAT4 $4^{-/-}$mice had similar amounts of granulocytes and mononuclear cells when compared with wildtype mice (Figure 10a). STAT6 ${ }^{-/-}$mice, however, had significantly higher numbers of granulocytes and mononuclear cells in liver. Similarly, lungs from wildtype mice and $\mathrm{STAT4}^{-/-}$mice had equivalent numbers of granulocytes and mononuclear cells, while STAT6 $6^{-1}$ mice had significantly higher numbers of these cells (Figure 10b). These observations support our MPO data and suggest that in addition to increased neutrophil accumulation, STAT6 $6^{--}$mice have augmented recruitment of mononuclear cells (most likely lymphocytes) to liver and lung 24 hours after endotoxin.

\section{Discussion}

STAT4 and STAT6 were originally defined as the critical transcription factors mediating Th1 and Th2 cell differentiation, respectively $(8,9)$. However, cytokines that activate STAT4 and STAT6, IL-12 and IL-13, have prominent roles in a wide variety of inflammatory responses, suggesting that STAT4 and STAT6 may have novel functions in these reactions. In the context of the systemic inflammatory response induced by endotoxin or sepsis, IL-12 and IL-13 appear to play contrasting roles. IL-12 has been shown to propagate the inflammatory response to endotoxin by augmenting proinflammatory cytokine production and contributing to endotoxin-induced death (10). Similarly, we have shown that IL-12 is a primary mediator of the hepatic inflammatory response to ischemia/reperfusion (19). However, IL-12 is also important for resistance to infection, with a primary role in the clearance of bacteria $(10,12)$. Conversely, IL-13 plays similar roles in both endotoxemia and sepsis by downregulating the production of inflammatory cytokines and chemokines and reducing organ neutrophil recruitment $(16,17)$.

Others have demonstrated that neutralization of IL-12 prevents the lethal effects of endotoxin (10). Until now STAT4 has been thought to mediate all of the known effects of IL-12; accordingly, we expected STAT4 ${ }^{--}$mice to be protected against endotoxininduced death. However, we found that STAT4 ${ }^{-/-}$mice were not protected from endotoxin-induced death but were much more susceptible to death than wild-type mice. We observed significantly more neutrophils (measured by MPO) in the lungs of STAT4 ${ }^{-/}$mice shortly after endotoxin (1-4 hours), but these changes were not accompanied by any noticeable differences in lung histopathology after 24 hours. These data are particularly interesting since they suggest that STAT4 activation by IL-12 has a beneficial effect. Furthermore, we found that blockade of IL-12 in STAT4 ${ }^{-/-}$mice completely abrogated the lethal effects of endotoxin. This suggests that
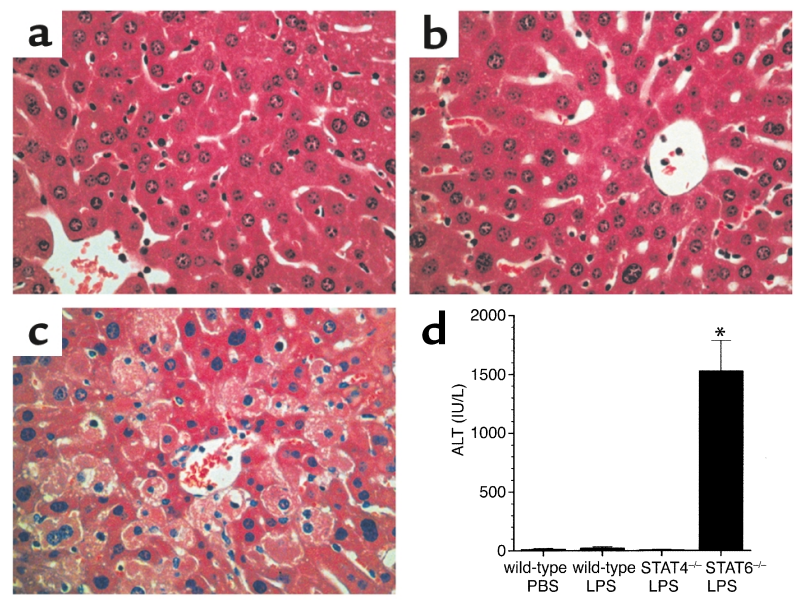

\section{Figure 8}

Endotoxemia causes hepatocellular injury in STAT6 ${ }^{-/-}$mice. Liver sections were taken from wild-type (a), STAT4 ${ }^{-1-}$ (b), or STAT6 ${ }^{-1-}$ (c) mice 24 hours after intraperitoneal injection of $3 \mathrm{mg} / \mathrm{kg}$ endotoxin. Livers from wild-type (a) and STAT4 ${ }^{-1-}$ (b) mice appeared relatively normal, while livers from STAT6 ${ }^{-1-}$ mice $(\mathbf{c})$ revealed vast areas of hepatocellular necrosis. Serum levels of ALT confirmed these histopathological observations (d). Serum was obtained 24 hours after the administration of endotoxin. Values are mean \pm SEM with $n=4$ mice per group. ${ }^{*} P<0.05$ compared with wild-type controls. 

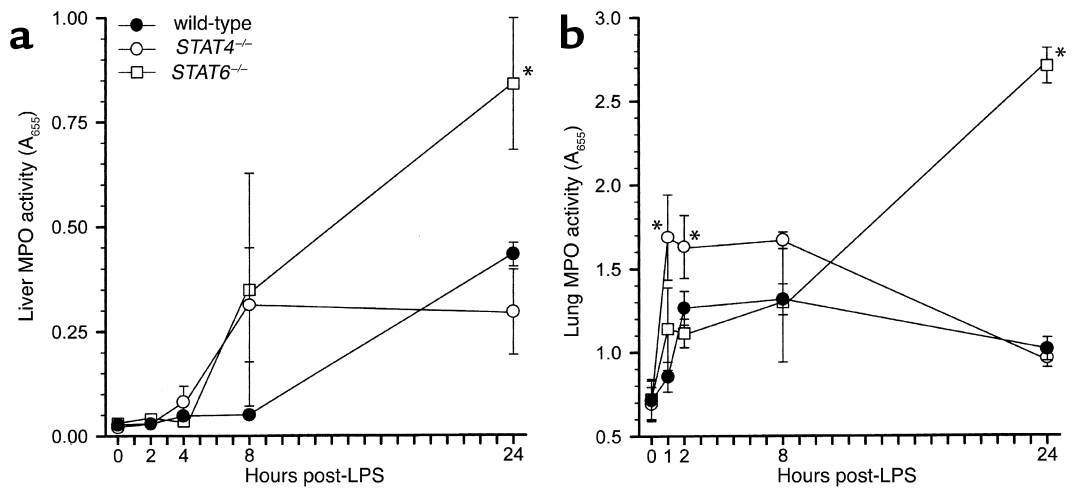

Figure 9

MPO activity in liver (a) and lung (b) tissues in wild-type, STAT4 ${ }^{-/-}$, and STAT6 ${ }^{-/-}$mice after intraperitoneal injection of $3 \mathrm{mg} / \mathrm{kg}$ endotoxin. Values are mean \pm SEM with $n=8-10$ mice per group. ${ }^{*} P<0.05$ compared with wild-type controls.

during endotoxemia IL-12 has divergent functions involving different signaling pathways. Supportive of this concept is the recent report that bacterial clearance after cecal ligation and puncture is similar in wild-type and STAT4 $4^{--}$mice (18). Blockade of IL-12 in the same model resulted in decreased bacterial clearance (12), suggesting that STAT4 does not mediate the antibacterial effects of IL-12. Together with our current findings, it appears that activation of STAT4 confers protection from endotoxin lethality while an as-yet undefined signaling pathway mediates other effects of IL- 12 .

In contrast to our current findings, Matsukawa et al. showed that STAT4 ${ }^{-/-}$mice were less susceptible to lethal septic peritonitis than their wild-type counterparts (18). This effect appeared to be due to increased organ expression of anti-inflammatory cytokines and reduced organ expression of CXC chemokines in STAT4 ${ }^{-/-}$mice. Subsequently, STAT4 ${ }^{-/}$mice had less neutrophil accumulation and organ injury (18). Here, in a model of endotoxemia, we found no differences in organ expression of cytokines or chemokines in wild-type and STAT4 ${ }^{-/-}$mice. These findings highlight the discrepancies between infectious (i.e., cecal ligation and puncture) and noninfectious (endotoxin) models of sepsis, which differ substantially in their complexity as well as in their inflammatory makeup (29). Histological assessment of all major organs (liver, lung, kidney, and heart) provided no further clues, as tissues from wild-type and STAT4-/mice were indistinguishable (data not shown). Thus, while it is clear that STAT4 ${ }^{-/-}$mice are more vulnerable to the lethal effects of endotoxin, the precise cause of this vulnerability remains an important question.

In wild-type mice, endotoxin induced an acute increase in STAT6 activation in lung, and more chronic activation was noted in both liver and lung. STAT6 $6^{-/}$mice were highly susceptible to the lethal effects of endotoxin. The mechanism of this increased mortality appears to be related to augmented expression of TNF- $\alpha$ and the chemokines MIP-2, IP-10, MIP-1 $\alpha$, and MCP- 1 in liver and lung tissues. The increased cytokine and chemokine production in $S T A T 6^{-/}$mice was associated with increased accumulation of neutrophils and mononuclear cells in liver and lung. In parallel, recent work from our laboratory has demonstrated that the activation of STAT6 reduces inflammatory liver injury induced by ischemia/reperfusion by suppressing the gene transcription of proinflammatory cytokines (30). Subsequently, others have shown that STAT6 suppresses NF-KB transcriptional activation by sequestering transcriptional coactivators (31). In the current studies, STAT6 ${ }^{--}$mice had greatly enhanced activation of NF- $\kappa B$ at 24 hours, suggesting that STAT6 plays a crucial role in the regulation of NF- $\mathrm{\kappa B}$ and subsequent production of proinflammatory mediators. These effects were manifested most notably in the liver. STAT6 ${ }^{-/-}$mice displayed a marked increase in the accumulation of both neutrophils and mononuclear cells in association with histological evidence of hepatocyte necrosis. Increased serum levels of ALT confirmed our histological observations. Together with our earlier findings in a model of liver ischemia/reperfusion $(30,32)$, these data provide strong evidence that STAT6 regulates systemic inflam-
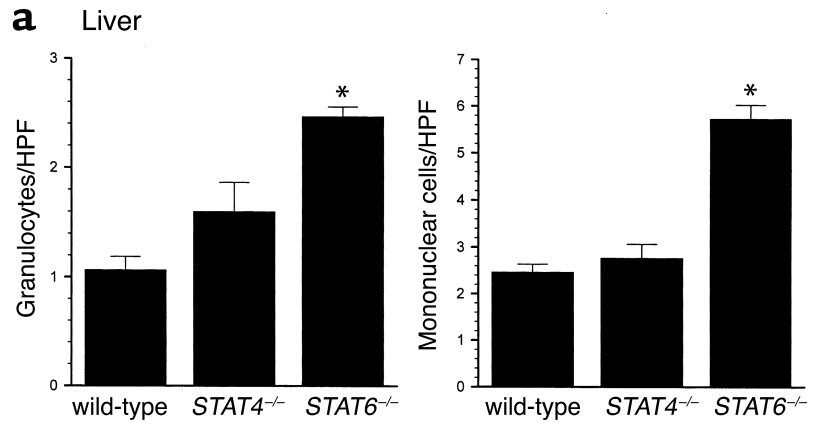

\section{b Lung}
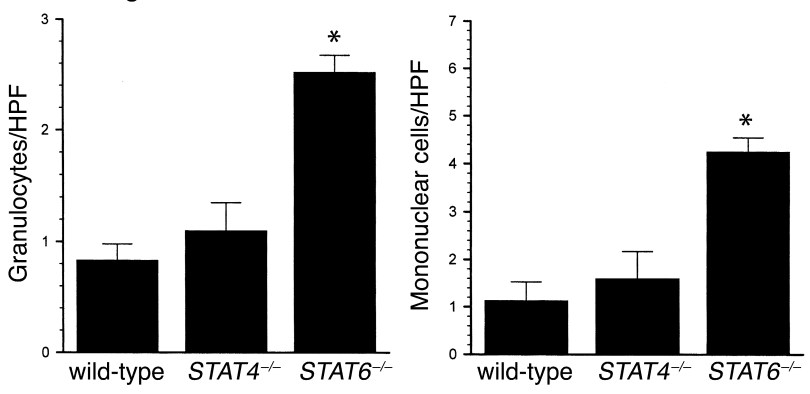

\section{Figure 10}

Recruitment of granulocytes and mononuclear cells to liver (a) and lung (b) after endotoxin. The number of granulocytes and mononuclear cells was counted in 20 high-power fields (HPFs) per tissue section. Values are mean \pm SEM with $n=3$ mice per group. ${ }^{*} P<0.05$ compared with wild-type controls. 
matory response to endotoxin by suppressing proinflammatory gene expression in multiple tissues.

The role of STAT6 in sepsis is more complicated. In a model of cecal ligation and puncture-induced peritonitis, $S T A T 6^{-1-}$ mice have increased peritoneal production of TNF- $\alpha$ and CC chemokines and increased neutrophil accumulation (18). These effects are quite similar to what we observed in liver and lung of STAT $6^{-/-}$mice after endotoxin administration. Following cecal ligation and puncture, the initial response of the host is to wall off the infection, and thus, front-line defenses are directed toward the peritoneum. In this respect, both the local inflammatory response to bacterial infection and the generalized systemic inflammation induced by endotoxin appear to be similarly augmented in the absence of STAT6. However, STAT6 ${ }^{-/-}$mice have reduced chemokine production and less neutrophil accumulation in distal organs (18). Furthermore, STAT6 ${ }^{-/-}$mice showed reduced mortality to the septic peritonitis. A possible explanation for these opposing effects in models of sepsis and endotoxemia may be attributable to the infectious burden. It appears that during septic peritonitis there is a differential cytokine response such that there is a local (peritoneal) Th1 response while a Th2 response is found in distal organs. As a result, alteration of the immune response by deletion of STAT 6 may be beneficial to host survival during infection, by allowing for a more effective antibacterial defense. Conversely, enhancement of the inflammatory response observed in $S T A T 6^{-/-}$during sterile endotoxemia may be detrimental to host survival.

Our studies provide new insights regarding the roles of STAT4 and STAT6 in a noninfectious model of systemic inflammation. Both STAT4 and STAT6 were found to function in a protective manner, albeit via divergent mechanisms. Perhaps the most significant finding of our study is that IL-12 appears to utilize multiple signaling pathways. STAT4, which previously was thought to be the primary transcription factor induced by IL-12, confers protective effects while an unknown pathway mediates other actions of IL-12, which may include induction of proinflammatory cascades and antibacterial defenses. STAT6, on the other hand, appears to regulate proinflammatory cytokine and chemokine expression by preventing activation of NF- $\mathrm{KB}$. These studies suggest that manipulation of the function of these STAT factors may provide substantial therapeutic benefit in a number of systemic and compartmentalized inflammatory disorders.

\section{Acknowledgments}

This work was supported in part by NIH grant DK-56029.

\footnotetext{
1. Guha, M., and Mackman, N. 2001. LPS induction of gene expression in human monocytes. Cell. Signal. 13:85-94.

2. Ghosh, S., May, M.J., and Kopp, E.B. 1998. NF- $\kappa$ B and Rel proteins: evolutionarily conserved mediators of immune responses. Annu. Rev. Immunol. 16:225-260.

3. Pahl, H.L. 1999. Activators and target genes of Rel/NF- $\mathrm{KB}$ transcription factors. Oncogene. 18:6853-6866.

4. Hack, C.E., Aarden, L.A., and Thijs, L.G. 1997. Role of cytokines in sepsis. Adv. Immunol. 66:101-195.

5. Martich, G.D., Boujoukos, A.J., and Suffredini, A.F. 1993. Response of man to endotoxin. Immunobiology. 187:403-416.
}

6. Christman, J.W., Lancaster, L.H., and Blackwell, T.S. 1998. Nuclear factor $\mathrm{\kappa B}$ : a pivotal role in the systemic inflammatory response syndrome and new target for therapy. Intensive Care Med. 24:1129-1130.

7. Takeda, K., and Akira, S. 2000. STAT family of transcription factors in cytokine-mediated biological responses. Cytokine Growth Factor Rev. 11:199-207.

8. Kaplan, M.H., Sun, Y.-L., Hoey, T., and Grusby, M.J. 1996. Impaired IL12 responses and enhanced development of Th2 cells in STAT4-deficient mice. Nature. 382:174-177.

9. Thierfelder, W.E., et al. 1996. Requirement for Stat4 in interleukin-12mediated responses of natural killer and T cells. Nature. 382:171-174

10. Zisman, D.A., et al. 1997. Anti-interleukin-12 therapy protects mice in lethal endotoxemia but impairs bacterial clearance in murine Escherichia coli peritoneal sepsis. Shock. 8:349-356.

11. Wysocka, M., et al. 1995. Interleukin-12 is required for interferongamma production and lethality in lipopolysaccharide-induced shock in mice. Eur. J. Immunol. 25:672-676.

12. Steinhauser, M.L., et al. 1999. Multiple roles for IL-12 in a model of acute septic peritonitis. J. Immunol. 162:5437-5443.

13. Kaplan, M.H., Schindler, U., Smiley, S.T., and Grusby, M.J. 1996. Stat6 is required for mediating responses to IL-4 and for development of Th2 cells. Immunity. 4:313-319.

14. Takeda, K., et al. 1996. Essential role of STAT6 in IL-4 signaling. Nature. 380:627-630.

15. Takeda, K., Kamanaka, M., Tanaka, T., Kishimoto, T., and Akira, S. 1996. Impaired IL-13-mediated functions of macrophages in STAT6-deficient mice. J. Immunol. 157:3220-3222.

16. Muchamuel, T., Menon, S., Pisacane, P., Howard, M.C., and Cockayne, D.A. 1997. IL-13 protects mice from lipopolysaccharide-induced lethal endotoxemia: correlation with down-modulation of TNF-alpha, IFNgamma, and IL-12 production. J. Immunol. 158:2898-2903.

17. Matsukawa, A., et al. 2000. Expression and contribution of endogenous IL-13 in an experimental model of sepsis. J. Immunol. 164:2738-2744.

18. Matsukawa, A., Kaplan, M.H., Hogaboam, C.M., Lukacs, N.W., and Kunkel, S.L. 2001. Pivotal role of signal transducer and activator of transcription (Stat) 4 and Stat 6 in the innate immune response during sepsis. J. Exp. Med. 193:679-688.

19. Lentsch, A.B., et al. 1999. Requirement for interleukin-12 in the pathogenesis of warm hepatic ischemia/reperfusion injury in mice. Hepatology. 30:1448-1453.

20. Deryckere, F., and Gannon, F. 1994. A one-hour minipreparation technique for extraction of DNA-binding proteins from animal tissues. Biotechniques. 16:405.

21. Villavedra, M., Carol, H., Hjulstrom, M., Holmgren, J., and Czerkinsky, C. 1997. "PERFEXT": a direct method for quantitative assessment of cytokine production in vivo at the local level. Res. Immunol. 148:257-266.

22. Schierwagen, C., Bylund-Fellenius, A.C., and Lundberg, C. 1990. Improved method for quantification of tissue PMN accumulation measured by myeloperoxidase activity. J. Pharmacol. Methods. 23:179-186.

23. Standiford, T.J., et al. 1995. Macrophage inflammatory protein-1 alpha mediates lung leukocyte recruitment, lung capillary leak, and early mortality in murine endotoxemia. J. Immunol. 155:1515-1524.

24. Zisman, D.A., et al. 1997. MCP-1 protects mice in lethal endotoxemia. J. Clin. Invest. 99:2832-2836.

25. Rovai, L.E., Herschman, H.R., and Smith, J.B. 1998. The murine neutrophil-chemoattractant chemokines LIX, KC, and MIP-2 have distinct induction kinetics, tissue distributions, and tissue-specific sensitivities to glucocorticoid regulation in endotoxemia. J. Leukoc. Biol. 64:494-502.

26. Frangogiannis, N.G., Mendoza, L.H., Smith, C.W., Michael, L.H., and Entman, M.L. 2000. Induction of the synthesis of the C-X-C chemokine interferon-gamma-inducible protein-10 in experimental canine endotoxemia. Cell Tissue Res. 302:365-376.

27. Bohrer, H., et al. 1997. Role of NFKB in the mortality of sepsis. J. Clin. Invest. 100:972-985.

28. Ruetten, H., and Thiemermann, C. 1997. Effect of calpain inhibitor I, an inhibitor of the proteolysis of I kappa B, on the circulatory failure and multiple organ dysfunction caused by endotoxin in the rat. Br. J. Pharmacol. 121:695-704.

29. Remick, D.G., Newcomb, D.E., Bolgos, G.L., and Call, D.R. 2000. Comparison of the mortality and inflammatory response of two models of sepsis: lipopolysaccharide vs. cecal ligation and puncture. Shock. 13:110-116.

30. Kato, A., Yoshidome, H., Edwards, M.J., and Lentsch, A.B. 2000. Regulation of liver inflammatory injury by signal transducer and activator of transcription-6. Am. J. Pathol. 157:297-302.

31. Ohmori, Y., and Hamilton, T.A. 2000. Interleukin-4/STAT6 represses STAT1 and NF- $\mathrm{KB}-$ dependent transcription through distinct mechanisms. J. Biol. Chem. 275:38095-38103.

32. Yoshidome, H., Kato, A., Miyazaki, M., Edwards, M.J., and Lentsch, A.B. 1999. IL-13 activates STAT6 and inhibits liver injury induced by ischemia/reperfusion. Am. J. Pathol. 155:1059-1064. 